\title{
Elasto-optic behaviour in epitaxial films of perovskite oxide ferroelectrics
}

\author{
Alexandr Dejneka ${ }^{\mathrm{a}}$ and Marina Tyunina $\mathrm{a}^{\mathrm{a}, \mathrm{b}}$

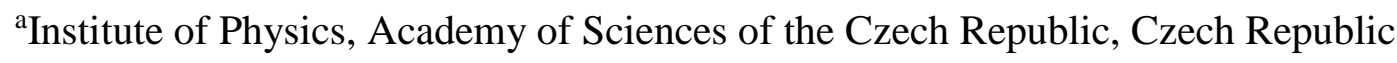 \\ ${ }^{b}$ Faculty of Information Technology and Electrical Engineering, Microelectronics Research Unit, \\ University of Oulu, Oulu, Finland
}

Large variations of refractive index in the visible spectral range are obtained in epitaxial perovskite oxide ferroelectric films experiencing lattice strain. The strain is imposed by substrates, on top of which the films are grown. The optical constants are determined using the spectroscopic ellipsometry. As a reference and for comparison, also prototype single crystals are inspected. The variations in refraction are related to the lattice strain in the films. Elasto-optic coefficient is formally estimated using the out-of-plane lattice elongation or shrinkage in the films compared to bulk. The obtained elasto-optic coefficients exceed significantly those previously reported for ferroelectric materials.

\section{Introduction}

Perovskite oxide $\mathrm{ABO}_{3}$ ferroelectrics are known for their excellent piezoelectric properties enabling numerous applications [1,2]. Strong piezoelectric effects coexist with remarkable optical behaviour in these materials. Ferroelectrics are wide bandgap insulators exhibiting high transparency and large refractive index $\mathrm{n}$ in the visible spectral range of radiation, namely: at photon energies of (1.6-3.1) $\mathrm{eV}$ or wavelengths of (400-780) $\mathrm{nm}$. Moreover, the refraction index $\mathrm{n}$ significantly changes under applied electric or stress fields. The phenomena are known as electro-optic and elasto-optic effects, correspondingly. The elasto-optic effect is employed in acousto-optic applications, where bulk singlecrystal materials are used. The achieved device performance is limited by the properties of available crystals, whose complicated growth technology restricts the materials selection. Besides difficulties in synthesis, also integration of crystals into modern systems is difficult. Today, remarkable progress is achieved in growth of ferroelectric thin films, including high-quality single-crystal-type epitaxial ones. Using thin films instead of crystals allows for significant expansion of materials selection and enables integrated optical applications [1,3-5]. As was shown in the last decades, the phase diagrams 
and ferroelectric properties of epitaxial films depend strongly on lattice strain therein. Studies of effects of epitaxy on optical behavior are at their beginning only [6-9]. Interestingly, an unexpectedly large effective elasto-optic coefficient has been recently reported in epitaxially strained $\mathrm{BiFeO}_{3}$ thin films [9]. Relationships between epitaxial strain and refractive index are practically unstudied and their understanding is missing. Here we experimentally investigate elasto-optic behaviour in epitaxial films of representative perovskite oxide ferroelectrics: $\mathrm{SrTiO}_{3}, \mathrm{NaNbO}_{3}, \mathrm{BaTiO}_{3}$, and $\mathrm{K}_{0.5} \mathrm{Na}_{0.5} \mathrm{NbO}_{3}$. The room-temperature state of unstressed $\mathrm{SrTiO}_{3}$ and $\mathrm{NaNbO}_{3}$ is non-ferroelectric: it is paraelectric in $\mathrm{SrTiO}_{3}$ and anti-ferroelectric in $\mathrm{NaNbO}_{3}$. The room-temperature state is ferroelectric in both $\mathrm{BaTiO}_{3}$ and $\mathrm{K}_{0.5} \mathrm{Na}_{0.5} \mathrm{NbO}_{3}$. The thin films were grown on different single-crystal substrates ensuring strain variations. As a reference and for comparison, also single crystals of $\mathrm{SrTiO}_{3}, \mathrm{BaTiO}_{3}$, and $\mathrm{NaNbO}_{3}$ were inspected.

\section{Experiment}

Cube-on-cube-type perovskite epitaxial films of $\mathrm{SrTiO}_{3}, \mathrm{BaTiO}_{3}, \mathrm{~K}_{0.5} \mathrm{Na}_{0.5} \mathrm{NbO}_{3}$, and $\mathrm{NaNbO}_{3}$ were grown by pulsed laser deposition using $\mathrm{SrTiO}_{3}(001), \mathrm{DyScO}_{3}(011), \mathrm{LaAlO}_{3}(001), \mathrm{LSAT}(001)$, and $\mathrm{KTaO}_{3}(001)$ substrates. The crystal structure of the films was studied by X-ray diffraction analysis and high-resolution transmission electron microscopy. The detailed analysis of the crystal phases, epitaxial relationships, and lattice strains were reported elsewhere [e.g. 6,7,10,11]. In order to estimate effect of strain on refractive index, the crystal structure of the films and prototype crystals was approximated by a pseudocubic perovskite, where the unit cell has the lattice parameters $a, b$, and c. The in-plane (parallel to the substrate surface) lattice parameters of the films are $a=b$, and the out-of-plane parameter is c. The out-of-plane strain in the film is calculated with the reference to the cubic or tetragonal-like c-parameter of the bulk prototype: $s=c_{\text {film }} / c_{\text {bulk }}-1$. For example, the strain is $\mathrm{s}=(3.846 / 3.905-1)=10.5 \times 10^{-3}$ in the $\mathrm{SrTiO}_{3}$ film on $\mathrm{KTaO}_{3}$ substrate. The calculated strain values for our films grown on the different substrates are presented in Table 1.

The optical constants of the films were determined using the spectroscopic ellipsometry. The ellipsometric spectra were measured on a J.A. Woollam variable angle spectroscopic ellipsometer, operating at photon energies of $(0.75-9) \mathrm{eV}$. The optical constants of the films were extracted from the measured data using a commercial WVASE32 software package [12]. The modelling procedure was initiated by constructing a four-phase model, which included a semi-infinite substrate, one homogeneous film, surface roughness, and ambient air. The film thickness for initial fitting procedure was taken form XRD measurements. The surface roughness for each sample was calculated as a mixture of $50 \%$ solid and $50 \%$ voids, as prescribed by the Bruggeman effective medium 
approximation [13]. The complex dielectric function $\varepsilon=\varepsilon_{1}+i \varepsilon_{2}$ was presented in a multi-oscillator form using $m$ Gaussian oscillators

$$
\varepsilon_{2 \mathrm{n}}=A_{\mathrm{m}} e^{-\frac{E-E_{\mathrm{m}}}{\mathrm{Br}}}-A_{\mathrm{m}} e^{-\frac{E+E_{\mathrm{m}}}{\mathrm{Br}_{\mathrm{mn}}}}
$$

where $A_{m}$ is the amplitude, $\mathrm{Br}_{\mathrm{m}}$ is the broadening (FWHM), and $E_{m}$ is the central energy of the oscillator. The oscillator parameters and layers' thicknesses were used as fitting variables. In the fitting procedure, all fitting parameters were determined simultaneously over the entire spectral range using Levenberg-Marquardt algorithm [14]. The refractive index and absorption coefficient were calculated from the fitted dielectric functions.

\section{Results}

The measured absorption spectra show that all films and crystals are transparent in the range of photon energies $E<3 \mathrm{eV}$. The refractive index was inspected in details in the range of $E=(1.0-3.5) \mathrm{eV}$. The films and crystal of $\mathrm{SrTiO}_{3}$ exhibit the refractive index $n \approx 2.3-2.8$ over the measured spectral range, with an increase at higher photon energies (shorter wavelength) (Figure 1(a)). Such dispersion of refraction is typical for perovskite oxide ferroelectric materials. The index is clearly smaller in the films compared to that in the crystal. The epitaxy-induced change is $\Delta \mathrm{n} \approx 0.05$ at $\mathrm{E}=2.0 \mathrm{eV}$. The index $\mathrm{n}$ at $\mathrm{E}=(1.0-2.5) \mathrm{eV}$ is practically insensitive to strain variations, while the changes become observable at higher energies, which are closer to the absorption edge. We note that unstressed $\mathrm{SrTiO}_{3}$ is paraelectric at room temperature. A strain-induced ferroelectricity has been achieved in epitaxial $\mathrm{SrTiO}_{3}$ films grown on $\mathrm{KTaO}_{3}$ substrates [15]. The observed decrease of $\mathrm{n}$ in the films compared to the crystal and the variations of $\mathrm{n}$ at $\mathrm{E}>2.5 \mathrm{eV}$ may be related to an onset of polar state in the films. Indeed, such an onset should result in the blue-shift of absorption edge and corresponding drop of $n$ [16]. Concurrently, epitaxial strain is expected to lead to a redshift of absorption edge in the paraelectric state [17]. The observed weak variations of $n$ in the films may be connected with the strain-polarization interplay therein.

Similarly to $\mathrm{SrTiO}_{3}$, also the refractive index of $\mathrm{NaNbO}_{3}$ is found to change in the films compared to that in crystal (Figure $1(\mathrm{~b}))$. The index in the films is clearly smaller: the change is $\Delta n \approx(0.07-$ 0.10) at $E=2.0 \mathrm{eV}$. Here, the strain-induced ferroelectric phase in the films contrasts with the antiferroelectric state of the crystal $[18,19]$. An epitaxially induced polarization leads to a drop of $\mathrm{n}$. The index remains practically unchanged with varying the sign and magnitude of strain in the films. 
Again, interplay between strain and strain-induced polarization may be responsible for the observed rather stable index in the films.

In contrast with the observations in $\mathrm{SrTiO}_{3}$ and $\mathrm{NaNbO}_{3}$, huge changes of refraction $\Delta \mathrm{n}$ in dependence on strain $s$ are found in the $\mathrm{BaTiO}_{3}$ and $\mathrm{K}_{0.5} \mathrm{Na}_{0.5} \mathrm{NbO}_{3}$ films. First, the decrease $\Delta \mathrm{n}$ in the epitaxial $\mathrm{BaTiO}_{3}$ films compared to refractive index in the polydomain $\mathrm{BaTiO}_{3}$ crystal is significantly larger than that observed in $\mathrm{SrTiO}_{3}$ and $\mathrm{NaNbO}_{3}$. The decrease is $\Delta \mathrm{n} \approx 0.17-0.35$ at $\mathrm{E}=2 \mathrm{eV}$ (Figure 2(a)). Furthermore, the change $\Delta n$ grows with increasing strain (out-of-plane elongation) in the $\mathrm{BaTiO}_{3}$ films. In order to quantify the correlation between the change $\Delta n$ of refraction and lattice strain $\mathrm{s}$, the refractive index was plotted as a function of strain at different photon energies $\mathrm{E}$ (Figure 2(b)). An effective elasto-optic coefficient $p^{*}$ was estimated as a slope of the linear fits to $\mathrm{n}^{-2} \propto s$.

The obtained coefficient is shown in Figure 2(c). The coefficient exhibits a minor dispersion in the visible spectral range of $E=1-2.5 \mathrm{eV}$. Remarkably, the coefficient is huge in this range, approximately $\mathrm{p}^{*} \approx 4.5-5$, which is an order of magnitude larger than the elasto-optic coefficient in the $\mathrm{BaTiO}_{3}$ bulk samples [20]. We note that the obtained gigantic $\mathrm{p}^{*}$ dramatically exceeds that reported in the epitaxial $\mathrm{BiFeO}_{3}$ films [9]. In order to cross-check the discovered unusual behaviour in the epitaxial $\mathrm{BaTiO}_{3}$ films, a batch of other ferroelectic films, $\mathrm{K}_{0.5} \mathrm{Na}_{0.5} \mathrm{NbO}_{3}$, was investigated. These studies demonstrate that the behavior found in $\mathrm{BaTiO}_{3}$ is not unique. The changes of similar character, but of even larger magnitude, are observed in the $\mathrm{K}_{0.5} \mathrm{Na}_{0.5} \mathrm{NbO}_{3}$ films (Figure 3). We stress that the obtained gigantic effective elasto-optic coefficient cannot be explained by possible inaccuracy in determining strain or refractive index. Indeed, the same experimental procedure revealed dramatically different types of optical behaviour in the films of non-ferroelectric perovskites $\left(\mathrm{SrTiO}_{3}\right.$ and $\left.\mathrm{NaNbO}_{3}\right)$ and in the films of ferroelectric perovskites $\left(\mathrm{BaTiO}_{3}\right.$ and $\left.\mathrm{K}_{0.5} \mathrm{Na}_{0.5} \mathrm{NbO}_{3}\right)$. We also note that the difference in behavior cannot be related to the types of cations in these materials. The only obvious difference is the presence or absence of ferroelectric polarization. As suggested by recent modeling [21], because of interaction of electro-magnetic wave of light with polar phonons, refractive index (far from absorption edge) may depend on temperature and polarization in ferroelectrics. Although such behavior qualitatively agrees with the earlier semi-empirical models [16], its mechanism is of another origin. Our experimental observations indicate that peculiar contribution of polar phonons may exist and lead to gigantic elasto-optic effects in epitaxial ferroelectric films. Further extensive experimental studies and theoretical modeling are highly desirable. 


\section{Conclusions}

Refractive index in the spectral range of (1-3.5) eV was experimentally studied in the cube-on-cubetype epitaxial films and prototype crystals of perovskite oxide paraelectric, antiferroelectric, and ferroelectric materials. The changes of index were inspected as a function of lattice strain. The moderate and strain-independent changes were found in the films of paraelectric $\mathrm{SrTiO}_{3}$ and antiferroelectric $\mathrm{NaNbO}_{3}$. The large and strain-dependent changes and the gigantic effective elastooptic coefficients were discovered in the films of ferroelectric $\mathrm{BaTiO}_{3}$ and $\mathrm{K}_{0.5} \mathrm{Na}_{0.5} \mathrm{NbO}_{3}$. The observed different types of behaviour are suggested to originate from the presence or absence of ferroelectric polarisation and associated polar phonons.

\section{Disclosure statement}

No potential conflict of interest was reported by the authors.

\section{Funding}

The authors acknowledge support from the Czech Science Foundation (Grantová Agentura České Republiky (GAČR)) (Grant Nos. 15-13778S and 15-15123S).

\section{References}

[1] Ferraro P, Grilli S, De Natale P. Ferroelectric crystals for photonic applications: including nanoscale fabrication and characterization techniques. Berlin: Springer Science \& Business Media; 2013.

[2] Lines M E, Glass A M. Principles and applications of ferroelectrics and related materials. Oxford: Oxford University Press; 1977.

[3] Qin M, Yao K, Liang YC. High efficient photovoltaics in nanoscaled ferroelectric thin films. Appl Phys Lett. 2008;93(12):122904.

[4] Guo R, You L, Zhou Y, et al. Non-volatile memory based on the ferroelectric photovoltaic effect. Nat Commun. 2013;4:1990.

[5] Dicken MJ, Sweatlock LA, Pacifici D, et al. Electrooptic modulation in thin film barium titanate plasmonic interferometers. Nano Lett. 2008;8:4048-4052.

[6] Tyunina M, Yao L, Chvostova D, et al. Concurrent bandgap narrowing and polarization enhancement in epitaxial ferroelectric nanofilms. Sci Technol Adv Mater. 2015;16:026002.

[7] Tyunina M, Chvostova D, Pacherova O, et al.Ambience sensitive optical refraction in ferroelectric nanofilms of $\mathrm{NaNbO}_{3}$. Sci Technol Adv Mater. 2014;15:045001. 
[8] Chernova E, Pacherova O, Chvostova D, et al. Strain controlled optical absorption in epitaxial ferroelectric $\mathrm{BaTiO}_{3}$ films. Appl Phys Lett. 2015;106:192903.

[9] Sando D, Yang Y, Bousquet E, et al. Large elasto-optic effect and reversible electrochromism in multiferroic $\mathrm{BiFeO}_{3}$. Nat Commun. 2016;7:10718.

[10] Dejneka A, Tyunina M, Narkilahti J, et al. Tensile strain induced changes in the optical spectra of $\mathrm{SrTiO}_{3}$ epitaxial thin films. Phys Solid State. 2010;52 (10):2082-2089.

[11] Chernova E, Pacherova O, Kocourek T, et al. Optical properties of ferroelectric epitaxial $\mathrm{K}_{0.5} \mathrm{Na}_{0.5} \mathrm{NbO}_{3}$ films in visible to ultraviolet range. PLoS ONE. 2016;11(4):e0153261.

[12] Woollam JA. WVASE software https://www.jawoollam.com/ellipsometry-software/ wvase

[13] Fujiwara H, Koh J, Rovira P I, et al. Assessment of effective-medium theories in the analysis of nucleation and microscopic surface roughness evolution for semiconductor thin films. Phys Rev B. 2000;61:10832-10844.

[14] Cardona M. Optical properties and band structure of $\mathrm{SrTiO}_{3}$ and $\mathrm{BaTiO}_{3}$. Phys Rev. 1965;140:A651-A655.

[15] Tyunina M, Narkilahti J, Plekh M, et al. Evidence for strain-induced ferroelectric order in epitaxial thin film $\mathrm{KTaO}_{3}$. Phys Rev Lett. 2010;104:260.

[16] Di Domenico Jr M, Wemple S H. Oxygen-octahedra ferroelectrics. I. Theory of electro-optical and nonlinear optical effects. J Appl Phys. 1969;40(2):720-734.

[17] Berger R , Fennie C J, Neaton J B. Band gap and edge engineering via ferroic distortion and anisotropic strain: the case of $\mathrm{SrTiO}_{3}$. Phys Rev Lett. 2011;107(14):2158.

[18] Lefkowitz I, Łukaszewicz K, Megaw H D. The hightemperature phases of sodium niobate and the nature of transitions in pseudosymmetric structures. Acta Crystallogr. 1966;20(5):670-683.

[19] Megaw HD. The seven phases of sodium niobate. Ferroelectrics. 1974;7(1):87-89.

[20] Lide David R. CRC handbook of chemistry and physics. 84th ed. London: CRC Press; 2003.

[21] Veithen M, Ghosez P. Temperature dependence of the electro-optic tensor and refractive indices of $\mathrm{BaTiO}_{3}$ from first principles. Phys Rev B. 2005;71(13):1751. 
Table 1. Strain values obtained for the various films on the different substrates.

\begin{tabular}{|l|c|l|c|}
\hline Film & Thickness, & Substrate & $\begin{array}{l}\text { Strain } \\
\text { value } \\
(\mathrm{s})\end{array}$ \\
\hline $\mathrm{SrTiO}_{3}$ & 10 & $\mathrm{DyScO}_{3}(011)$ & -5 \\
\hline $\mathrm{SrTiO}_{3}$ & 10 & $\mathrm{KTaO}_{3}(001)$ & -10 \\
\hline $\mathrm{NaNbO}_{3}$ & 10 & $\mathrm{SrTiO}_{3}(001)$ & +2 \\
\hline $\mathrm{NaNbO}_{3}$ & 10 & $\mathrm{DyScO}_{3}(011)$ & -11 \\
\hline $\mathrm{NaNbO}_{3}$ & 10 & $\mathrm{LSAT}(001)$ & +6.6 \\
\hline $\mathrm{BaTiO}_{3}$ & 8 & $\mathrm{SrTiO}_{3}(001)$ & +13 \\
\hline $\mathrm{BaTiO}_{3}$ & 11 & $\mathrm{SrTiO}_{3}(001)$ & +8 \\
\hline $\mathrm{BaTiO}_{3}$ & 20 & $\mathrm{SrTiO}_{3}(001)$ & +3 \\
\hline $\mathrm{K}_{0.5} \mathrm{Na}_{0.5} \mathrm{NbO}_{3}$ & 10 & SrTiO $_{3}(001)$ & +12.5 \\
\hline $\mathrm{K}_{0.5} \mathrm{Na}_{0.5} \mathrm{NbO}_{3}$ & 10 & $\operatorname{DyScO}_{3}(011)$ & +7 \\
\hline $\mathrm{K}_{0.5} \mathrm{Na}_{0.5} \mathrm{NbO}_{3}$ & 10 & $\mathrm{LSAT}(001)$ & 0 \\
& & & \\
\hline
\end{tabular}



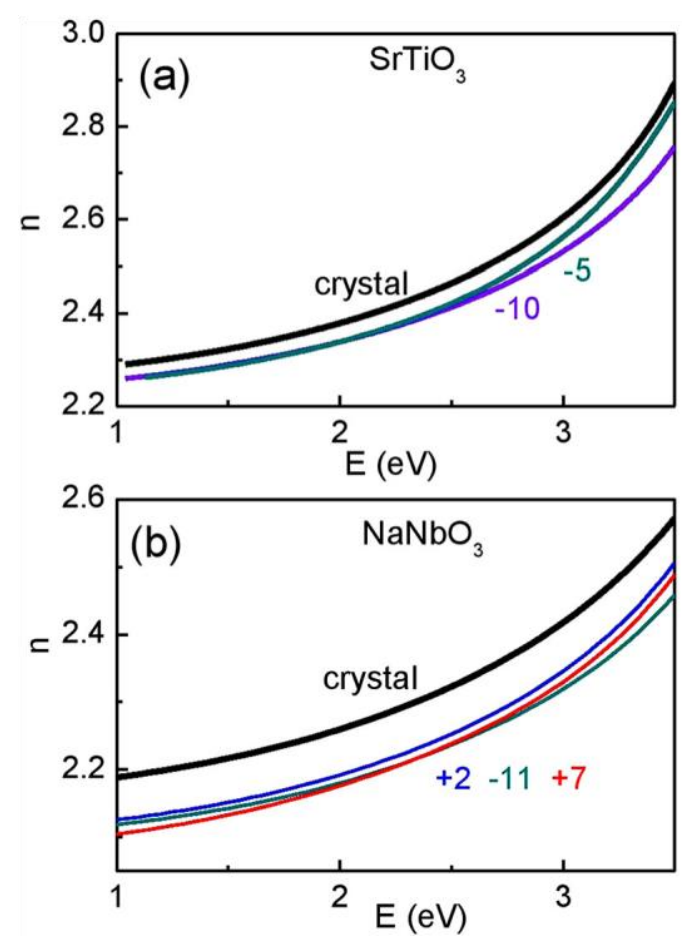

Figure 1. (a, b) Refractive index as a function of photon energy in (a) $\mathrm{SrTiO}_{3}$ and (b) $\mathrm{NaNbO}_{3}$. Lattice strains $\mathrm{s}$ in the films are marked in the plots as numbers colored as the appropriate lines. 

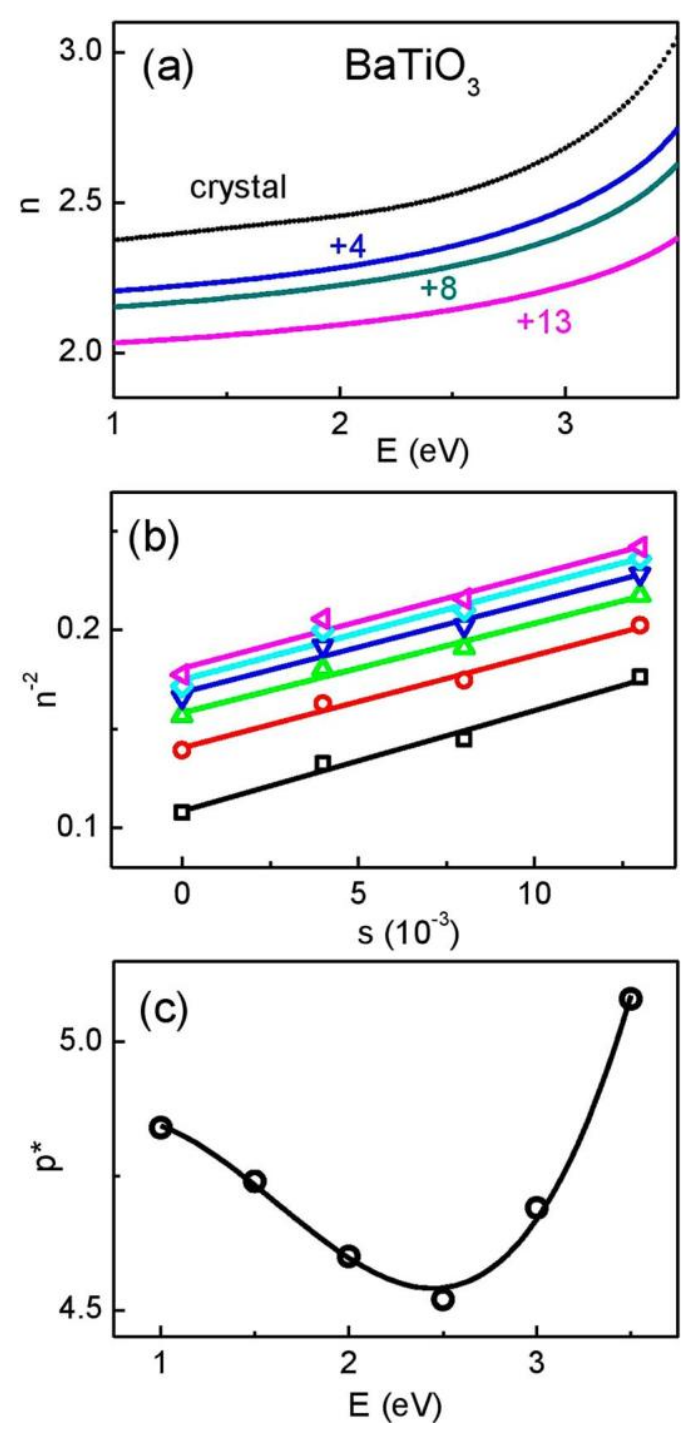

Figure 2. BaTiO3. (a) Refractive index $\mathrm{n}$ as a function of photon energy at different lattice strains. (b) Inverse squared of refractive index n-2 as a function of lattice strain at different photon energies. Solid lines show fits in (b). Lattice strains $s$ in the films are marked in the plots as numbers coloured as the appropriate lines in (a). The energy is $\mathrm{E}=1,1.5,2,2.5,3$, and $3.5 \mathrm{eV}$ from the upper curve down in (b). (c) Effective elasto-optic coefficient p* as a function of photon energy in the $\mathrm{BaTiO}_{3}$ films. 

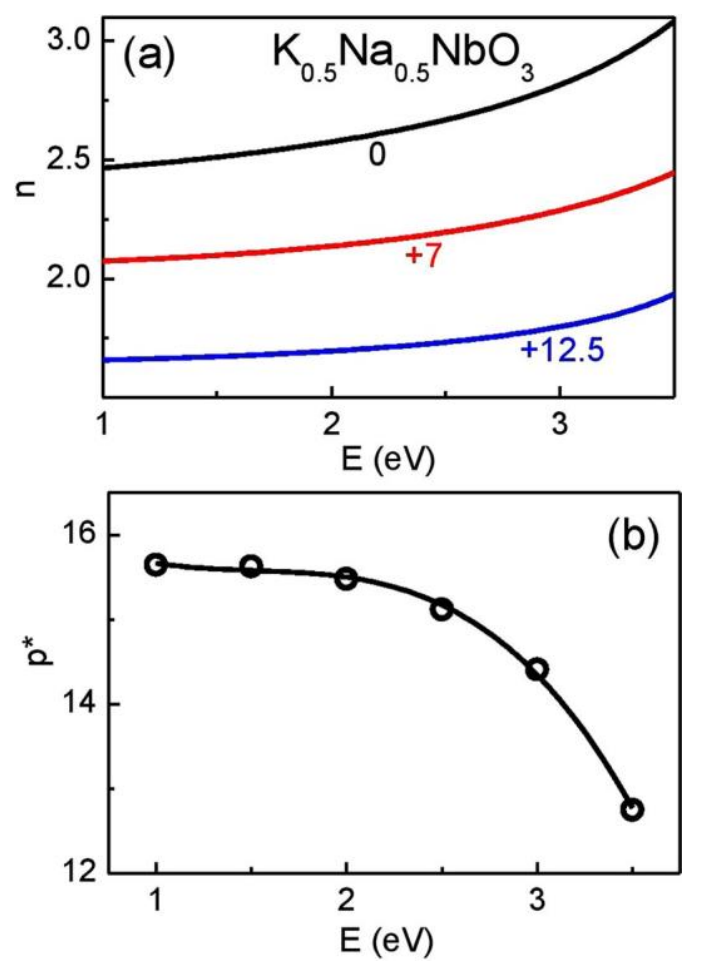

Figure 3. $\mathrm{K}_{0.5} \mathrm{Na}_{0.5} \mathrm{NbO}_{3}$ (a) refractive index as a function of photon energy at different lattice strains. Lattice strains $s$ in the films are marked in the plots as numbers coloured as the appropriate lines. (b) Effective elasto-optic coefficient $\mathrm{p}^{*}$ as a function of photon energy in the $\mathrm{K}_{0.5} \mathrm{Na}_{0.5} \mathrm{NbO}_{3}$ films. 